\title{
RESEARCH
}

Open Access

\section{The within-subject application of diffusion tensor MRI and CLARITY reveals brain structural changes in Nrxn2 deletion mice}

Eleftheria Pervolaraki ${ }^{1+}$, Adam L. Tyson ${ }^{2,3,4+}$, Francesca Pibiri ${ }^{5}$, Steven L. Poulter ${ }^{5}$, Amy C. Reichelt ${ }^{6}$, R. John Rodgers ${ }^{7}$, Steven J. Clapcote ${ }^{1}$, Colin Lever ${ }^{5}$, Laura C. Andreae ${ }^{2,3+}$ and James Dachtler ${ }^{1,5^{*}+}$ (i)

\begin{abstract}
Background: Of the many genetic mutations known to increase the risk of autism spectrum disorder, a large proportion cluster upon synaptic proteins. One such family of presynaptic proteins are the neurexins (NRXN), and recent genetic and mouse evidence has suggested a causative role for NRXN2 in generating altered social behaviours. Autism has been conceptualised as a disorder of atypical connectivity, yet how single-gene mutations affect such connectivity remains under-explored. To attempt to address this, we have developed a quantitative analysis of microstructure and structural connectivity leveraging diffusion tensor MRI (DTI) with high-resolution 3D imaging in optically cleared (CLARITY) brain tissue in the same mouse, applied here to the Nrxn2a knockout (KO) model.
\end{abstract}

Methods: Fixed brains of Nrxn2a KO mice underwent DTI using 9.4T MRI, and diffusion properties of socially relevant brain regions were quantified. The same tissue was then subjected to CLARITY to immunolabel axons and cell bodies, which were also quantified.

Results: DTI revealed increases in fractional anisotropy in the amygdala (including the basolateral nuclei), the anterior cingulate cortex, the orbitofrontal cortex and the hippocampus. Axial diffusivity of the anterior cingulate cortex and orbitofrontal cortex was significantly increased in Nrxn2a KO mice, as were tracts between the amygdala and the orbitofrontal cortex. Using CLARITY, we find significantly altered axonal orientation in the amygdala, orbitofrontal cortex and the anterior cingulate cortex, which was unrelated to cell density.

Conclusions: Our findings demonstrate that deleting a single neurexin gene (Nrxn2a) induces atypical structural connectivity within socially relevant brain regions. More generally, our combined within-subject DTI and CLARITY approach presents a new, more sensitive method of revealing hitherto undetectable differences in the autistic brain.

Keywords: MRI, CLARITY, Social, Autism, Axons, Diffusion, Structure, Imaging

\footnotetext{
* Correspondence: james.dachtler@durham.ac.uk

†Eleftheria Pervolaraki, Adam L. Tyson, Laura C. Andreae and James Dachtler contributed equally to this work.

${ }^{1}$ School of Biomedical Sciences, University of Leeds, Leeds LS2 9JT, UK

${ }^{5}$ Department of Psychology, Durham University, South Road, Durham DH1

3LE, UK

Full list of author information is available at the end of the article
}

(c) The Author(s). 2019 Open Access This article is distributed under the terms of the Creative Commons Attribution 4.0 International License (http://creativecommons.org/licenses/by/4.0/), which permits unrestricted use, distribution, and reproduction in any medium, provided you give appropriate credit to the original author(s) and the source, provide a link to the Creative Commons license, and indicate if changes were made. The Creative Commons Public Domain Dedication waiver (http://creativecommons.org/publicdomain/zero/1.0/) applies to the data made available in this article, unless otherwise stated. 


\section{Background}

Autism is a common neurodevelopmental disorder, which is highly heritable [1]. While heritability is high, it is also clear that autism is highly polygenic. Around $~ 400-1000$ genes are involved in autism susceptibility [2-5]. Many of these genes cluster upon proteins relating to synaptic signalling [6]. A family of presynaptic proteins garnering recent interest have been the neurexins (NRXNs). NRXNs are encoded by three genes (NRXN1, NRXN2, NRXN3; note that CNTNAP1 and CNTNAP2 are sometimes referred to as NRXN4), of which two major isoforms exist: the longer $\alpha$ proteins with six laminin/neurexin/sex hormone (LNS) binding domains, and the shorter $\beta$ proteins with one LNS binding domain $[7,8]$.

Mutations within all three NRXN genes have been linked to autism [6]. Heterozygous deletions within NRXN2 have been identified in a number of individuals with autistic phenotypes. These include an autistic boy and his father (who had severe language delay but not autism) who both had a frameshift mutation within exon 12 of NRXN2 [9]; a $570-\mathrm{kb}$ de novo deletion of 24 genes at chromosome 11q13.1, including NRXN2, in a 21-year-old man displaying a clinical phenotype including autistic traits [10]; a $1.6-\mathrm{Mb}$ deletion at chromosome region 11q12.3-11q13.1, including $N R X N 2$, in a 23-year-old man with intellectual disability and behavioural problems [11]; a de novo frameshift mutation identified in a Chinese man with autism spectrum disorder (ASD) [12], a $921-\mathrm{kb}$ microdeletion at $11 \mathrm{q} 13$ in a 2-year-old boy who had language and developmental delay (although did not meet the autism diagnosis criteria) [13] and a paternally inherited microRNA miR-873-5p variant in an ASD individual which altered binding affinity for several risk genes including NRXN2 and CNTNAP2 (NRXN4) [14]. Furthermore, recently, two large-scale reports have identified NRXN2 with ASD risk. A study of 529 ASD patients and 1923 controls in a Chinese population identified two NRXN2 variants which significantly increase ASD risk [15]. The second study employed machine learning approaches across 5000 ASD families to rank the importance of ASD candidate genes and ranks NRXN2 in the top $0.5 \%$ of genes, i.e. 113th [16]. For comparison, NRXN1, for which the evidence base for its links to ASD is broader and stronger, ranks 45 , and CNTNAP2 ranks 211th [16]. Consistent with these association studies, we and others have previously found that homozygous or heterozygous deletion of $N r x n 2 \alpha$ induces impairment in social approach and social recognition [17-19]. In summary, although mutations within NRXN2 are rare, understanding how they may drive social, ASD-relevant behavioural changes is important. One important goal is to help elucidate how apparently convergent pathophysiology in ASD emerges despite marked genetic heterogeneity [5]; mapping brain alterations driven by different single genes is thus a crucial task.
Currently, it is unknown whether deletion of $N r x n 2 \alpha$ changes the brain's microstructure and connectivity. One previous study found coarse alterations to cell layer thickness within the hippocampus of $N r x n 2 \alpha$ homozygous KOs [20]. However, cell density measurements are unlikely to reveal the true extent of changes within the autistic brain. Within the current study, we have addressed this by developing a dual imaging approach (DTI and CLARITY) that quantifies the alignment and density of white matter, applied here to brain regions known to support social behaviour in a mouse model of autism.

Diffusion tensor MRI (or DTI) is based upon the movement of water molecules, a measure that is termed fractional anisotropy (FA). Apparent diffusion coefficient (ADC) is similar to FA but quantifies diffusion restriction as opposed to the spatial symmetry of diffusion. This approach has been used to explore neuropathological markers in autistic patients; alterations in myelination, axonal abundance, size and orientation all modify FA and ADC values [21-23]. Using the preferred direction of the diffusion of tensors between brain regions can be used to explore their potential connection. Quantification of those computed streamlines by FA and axial and/or radial diffusion can indicate impairments in regional structural connectivity. Since aberrant brain connectivity is likely a core feature of autism [24], we reasoned that the candidate method for probing the autistic brain should combine tractographic techniques. Accordingly, here, we combined high-resolution imaging of labelled neuronal tracts in brains rendered transparent by CLARITY with DTI.

CLARITY is a recent development that renders tissue optically transparent and macromolecule permeable [25]. This permits antibody staining and imaging of much larger tissue volumes than possible under traditional immunofluorescence techniques. By examining fibre orientation without sectioning-related artefacts and biases, axonal staining in cleared tissue affords a deeper understanding of the microstructure and structural connectivity of a brain region.

Given the social impairments found within $N r x n 2 \alpha$ mice, we sought to examine those brain regions most closely linked with social behaviour (see Additional file 1: Supplemental materials). Briefly, we identified four regions of interest (ROIs): the amygdala and three brain regions strongly and directly connected to the amygdala; the hippocampus, orbitofrontal cortex (OFC), and anterior cingulate cortex (ACC). As predicted, structural connectivity was abnormal in $N r x n 2 \alpha$ mice.

\section{Methods}

\section{Ethics}

All procedures were approved by the University of Leeds and Durham University Animal Ethical and Welfare 
Review Boards and were performed under UK Home Office Project and Personal Licenses in accordance with the Animals (Scientific Procedures) Act 1986.

\section{Animals}

Full details of the animals, their background, genotyping and housing can be found elsewhere [17]. In brief, male B6;129-Nrxn3tm1Sud/Nrxn1tm1Sud/Nrxn2tm1Sud/J mice (JAX \#006377) were purchased from the Jackson Laboratory and outbred once to the C57BL/6 $\mathrm{NCrl}$ strain (Charles River, Margate, UK) to obtain mice that were individually $N r x n 2 \alpha \mathrm{KO}$ heterozygotes. Subsequently, HET knockout males were bred with HET females (cousin mating).

\section{Experimental animals}

Six adult wild-type males (Charles River, Margate, UK) and six age-matched littermate $N r \times n 2 \alpha$ KO homozygotes (71 days \pm 6 days old (SEM)) were perfused fixed with $4 \%$ paraformaldehyde (PFA) in $0.1 \mathrm{M}$ phosphate buffer saline (PBS) and the brains extracted. The brains were immersed in 4\% PFA/0.1 M PBS for a minimum of $48 \mathrm{~h}$ prior to imaging. Mouse weights were not specifically taken prior to perfusion. However, in a separate cohort, wild-type and Nrxn2 $\alpha$ KO homozygotes did not significantly differ in body mass (wild type, $n=15,30.9$ $\pm 4.1 \mathrm{~g} ; \mathrm{Nrxn} 2 \mathrm{KO}, n=10,28.6 \pm 4.3 \mathrm{~g}, t$ test $p=0.167$ ). We did not specifically time perfusions, although as a matter of process, each mouse was perfused with $\sim 60$ $\mathrm{ml}$ of fixative. We cannot rule out that variance in perfusion timings may have influenced the results, which is a limitation of the current study. During imaging, the samples were placed in custom-built MR-compatible tubes containing Fomblin Y (Sigma, Poole, Dorset, UK).

Due to the relatively low variance and owing to the complexity and methodological nature in our experimental approach, we achieved significance by groups of 6 (power provided in the 'Results' section). No data was excluded from the study. Sample randomisation was performed by JD, with experimenters (EP and ALT) blinded to genotype.

\section{Data acquisition}

Image acquisition has been described elsewhere [26]. Each brain was 3D imaged using the protocol TE $35 \mathrm{~ms}$, TR $700 \mathrm{~ms}$ and 10 signal averages. The field of view was set at $128 \times 128 \times 128$, with a cubic resolution of $100 \mu \mathrm{m} /$ pixel and a $b$ value of $1200 \mathrm{~s} / \mathrm{mm}^{2}$. Further details can be found in Additional file 1: Supplemental materials.

\section{Image processing}

Parsing of the raw data was semi-automated using DSI Studio, in order to obtain $b$ values for every normalised gradient vector on the $x, y$ and $z$ orientations. Unwanted background, setting a threshold, smoothing of the data and definition of tissue boundaries were performed prior to the reconstruction of the final 3D image. DTI analysis parameters were calculated as previously described [27].

The ex vivo mouse brain 3D diffusion-weighted images were reconstructed from the Bruker binary file using DSI Studio (http://dsi-studio.labsolver.org) [28]. Direction Encoded Colour Map (DEC) images were generated by combining the information from the primary eigenvectors, diffusion images and the FA. Images of the primary vectors and their orientation were reconstructed and superimposed on the corresponding FA images to guide the segmentation of discrete anatomical locations according to the brain atlas (Fig. 1b-d). Region of interest definition was performed by author EP and corroborated independently by JD, with region area compared between the experimenters (data not shown). For whole brain region analysis, we used a similar approach, except regions were segmented for every other slice in the anterior to posterior extent (Fig. 1a-d; Additional file 1: Figure S1) [29]. The DSI Studio DTI reconstruction characterises the major diffusion direction of the fibre within the brain [30,31]. Extraction of FA (calculated [26]) and ADC was performed within selected segmented brain areas for every 3D-reconstructed mouse brain.

\section{Regions of interest (ROIs)}

Our DTI approach was to undertake an a posteriori analysis of neural organisation in regions of interest (ROIs) identified by previous literature as socially relevant. Given the social impairments found within $N r x n 2 \alpha$ mice, for the current study, we identified the brain regions of interest (ROIs) most closely linked with social behaviour, using previously published reports of brain region involvement in social behaviour. Quantification of c-Fos immunoreactivity has highlighted the importance of several amygdala nuclei (including the basolateral) following social exposure [32], but also the anterior cingulate cortex (ACC), prefrontal cortex and the hippocampus [33]. Lesions to the primate amygdala alter social interactions $[34,35]$, and amygdala neurons in primates including humans increase firing rates during social scenarios [36-38]. Consistent with these animal studies, amygdala damage in humans [39] and amygdala dysfunction in ASD patients [40] impair social responses. Other socially important brain regions have also been proposed. Notably, several studies have implicated the rodent hippocampus in social behaviour, including social memory and sociability [41-43]. For instance, intrahippocampal administration of neurolide-2, which interacts with $\alpha$-neurexin, specifically impairs sociability, but not anxiety and spatial learning in rats [44]. These findings are consistent with reports of social deficits in humans with hippocampal damage [45] and hippocampal 

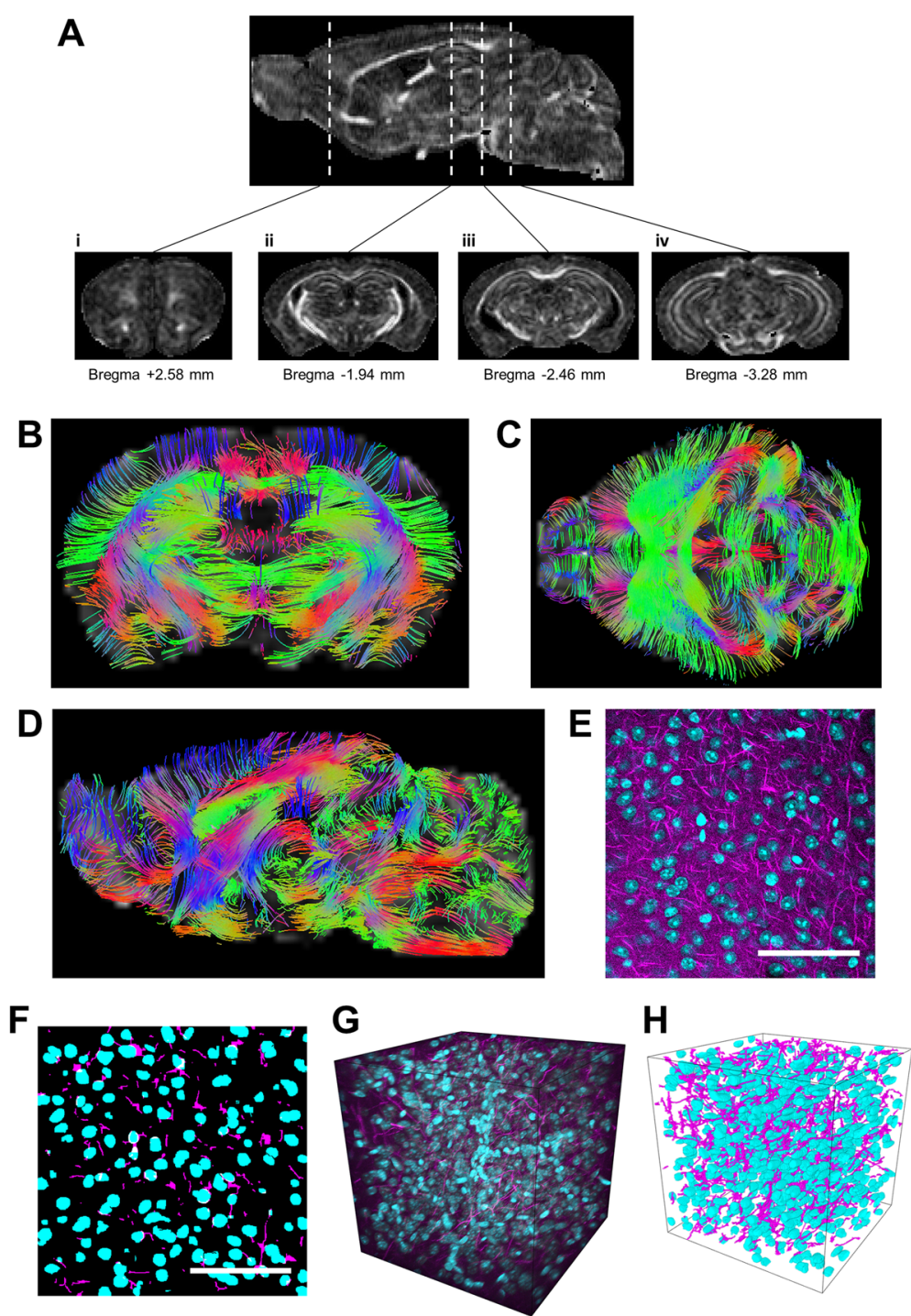

Fig. 1 Quantification of CLARITY imaging. a Sections of DTI-scanned brain were segmented at different bregma levels for (i) the orbitofrontal cortex, (ii) the anterior hippocampus and amygdala, (iii) the mid hippocampus and posterior amygdala and (iv) the posterior hippocampus. b-d DTI-scanned brains were computed for tracts. Tissue from wild-type and Nrxn2a KO mice were cleared and stained for neurofilament and DAPI (e). f Automated MATLAB scripts were used to segment the DAPI (blue) and neurofilament (purple) channels such that cell density and axonal density and orientation could be calculated. $\mathbf{g}$ is representative of a CLARITY-derived 3D stacked image of a DAPI and neurofilament of a region of interest, with $\mathbf{h}$ being the corresponding segmented image. Scale bar, $100 \mu \mathrm{m}$

abnormalities in ASD [46, 47]. Finally, several studies link the frontal cortex, particularly the orbitofrontal cortex, which is strongly anatomically connected with the amygdala [48], to social processing [49, 50], consistent with findings of abnormalities in orbitofrontal cortex in ASD [48, 51]. Control regions of the primary motor cortex (M1), primary sensory cortex (S1) and the barrel field were chosen for CLARITY (Additional file 1: Figure S7N-O).

\section{Clarity}

Following MR imaging, the brains were washed in PBS to remove all Fomblin $\mathrm{Y}$ and then incubated for 7 days in hydrogel solution at $4{ }^{\circ} \mathrm{C}$ prior to polymerisation at $37^{\circ} \mathrm{C}$ for $3.5 \mathrm{~h}$. The tissue was cut into $1.5-\mathrm{mm}$ coronal sections using a custom 3D-printed brain-slicing matrix based on MRI scans of an adult C57BL/6 mouse brain [52] and incubated in clearing buffer for 24 days at $37{ }^{\circ} \mathrm{C}$ with shaking. The cleared tissue was then washed in PBSTN $_{3}(0.1 \%$ TritonX-100 and $1.5 \mathrm{mM}$ sodium azide in $\mathrm{PBS}$ ) for $24 \mathrm{~h}$ at room temperature and incubated in primary antibody solution (neurofilament (Aves NF-H) 1:100 in $\mathrm{PBSTN}_{3}$ ) at $37^{\circ} \mathrm{C}$ with shaking for 13 days. Samples were washed and then incubated in secondary antibody (AlexaFluor 488 goat anti-chicken IgY) as per 
the primary. Sections were washed again and incubated in $3.6 \mu \mathrm{M}$ DAPI (4',6-diamidino-2-phenylindole) followed by $85 \%$ glycerol in PBS for refractive index matching.

Cleared samples were imaged using a Zeiss 7MP multiphoton microscope at $770 \mathrm{~nm}$ using a $20 \times$ objective lens (W Plan-Apochromat, NA 1.0, WD $1.7 \mathrm{~mm}$ ). Images $(512 \times 512 \times 512$ voxels or $265 \times 265 \times 265 \mu \mathrm{m}$ with an isotropic resolution of $520 \mathrm{~nm}$ ) were acquired in ACC, basolateral (BLA) and basomedial amygdala and OFC in both hemispheres. DAPI and neurofilament signal was segmented into cell nuclei and axons, and the resulting binary images were used to generate values for cell density, axonal density and axonal alignment.

Full CLARITY methodological details are available within Additional file 1: Supplemental materials.

\section{Data availability}

Codes to analyse CLARITY datasets are made available by author LCA by email request to either JD or LCA, subject to reference to the current paper. The datasets used and/or analysed during the current study are available from the corresponding author on reasonable request.

\section{Data analysis}

All data are expressed as mean \pm standard error of the mean (SEM). To assess the variance between genotypes within a single brain structure across hemispheres (given the importance of hemispheric differences in ASD [53]), data was analysed by within-subject repeated measures two-way ANOVAs, with Sidak multiple corrections employed on post hoc testing, or unpaired $t$ tests. To correct for multiple comparisons, we employed the Benjamini-Hochberg procedure (corrected $p$ values stated). Non-significant statistical results, particularly hemisphere comparisons, can be found in Additional file 1: Supplemental materials. Statistical testing and graphs were made using GraphPad Prism version 6 and SPSS v22.

\section{Results}

\section{Nrxn2a deletion disrupts DTI measures of microstructure} in social brain regions

To assess whether $N r \times n 2 \alpha$ deletion alters gross morphology, we quantified whole brain volume using DTI. We found total brain volume for wild types and $N r \times n 2 \alpha$ KOs was similar $\left(456.0 \pm 14.76\right.$ vs. $466.2 \pm 11.0 \mathrm{~mm}^{3}$ (respectively); $\left.t_{(10)}=0.55, p=0.59\right)$. Thus, Nrxn2 $\alpha$ deletion does not change the total brain size.

To quantitatively measure DTI, we examined FA and ADC. FA analyses changes in the linear orientation (i.e. along an axonal tract), whereas ADC (mean diffusivity) averages diffusion in all directions (i.e. the $X-, Y_{-}$and $Z$ - axis), which is sensitive to changes such as altered alignment. The amygdala is critically important for social behaviours. To assess whether amygdalar alterations might account for social impairments in Nrxn2 $\alpha \mathrm{KO}$ mice, we segmented the whole amygdala structure and the basolateral nuclei along the anterior-posterior axis.

The amygdala showed a significant increase in FA in $N r x n 2 \alpha$ KO mice (Fig. 2a) (genotype $\left(F_{(1,10)}=11.15, p=\right.$ 0.022 , power $=85.2 \%)$ ). There was a FA reduction observed specifically in the BLA, a region strongly associated with social behaviours (Fig. 2b; genotype $\left(F_{(1,10)}=\right.$ 6.31, $p=0.049)$ ). ADC was not significantly altered in the whole amygdala or BLA (Fig. 2c, d; all genotype $F_{(1}$, 10) $<1$ ).

We conducted the same analysis for the two prefrontal regions implicated in social behaviour and autism: the OFC and ACC. The pattern of results was similar for both regions: FA was significantly altered, while ADC was unaffected (Fig. 3a, b) and the ACC (Fig. 3e, f). FA for the OFC was significantly increased (genotype $\left(F_{(1}\right.$, 10) $=16.14, p=0.009$, power $=95.0 \%)$ ), but ADC was similar between the genotypes (genotype $\left(F_{(1,10)}=1.43\right.$, $p=0.11)$ ). The ACC also had significantly increased FA $\left(t_{(10)}=2.55, p=0.03\right.$, power $\left.=71.0 \%\right)$, but ADC was unaltered $\left(t_{(10)}=0.51, p=0.618\right)$.

We sought to examine whether changes in the amygdala, OFC or ACC FA and ADC were driven by diffusion in the primary axis $\left(\lambda_{1}\right)$ or the radial orientations $\left(\lambda_{2}\right.$ and $\left.\lambda_{3}\right)$ by characterisation of $\mathrm{AD}$ (primary) and $\mathrm{RD}$ (radial). Within the amygdala, neither $\mathrm{AD}$ nor $\mathrm{RD}$ was significantly altered in Nrxn2 $\alpha$ KO mice (Fig. 2e; AD genotype: $F_{(1,10)}=3.06$, $p=0.111$, Fig. 2f; RD genotype: $F_{(1,10)}=2.47, p=0.147$ ). Within the OFC (Fig. 3c, d), AD was significantly increased (genotype $\left(F_{(1,10)}=6.71, p=0.032\right.$, power $\left.=64.7 \%\right)$ ), whereas RD was significantly decreased (genotype $\left(F_{(1}\right.$, 10) $=10.07, p=0.025$, power $=81.5 \%)$ ), suggesting that both along-tract diffusion and tract branching were affected. However, in the ACC (Fig. 3g-h), only AD was significantly increased $\left(t_{(10)}=3.89, p=0.019\right.$, power $\left.=96.9 \%\right)$, with no alteration in $\mathrm{RD}\left(t_{(10)}=1.35, p=0.10\right)$. Increased $\mathrm{AD}$ and decreased $\mathrm{RD}$ is thought to reflect changes in axonal density or orientation [54].

\section{DTI reveals altered hippocampal microstructure in $\mathrm{Nrxn2a}$ KO mice}

The hippocampus has recently been associated with social motivation and social recognition. Since the specific contributions of the dorsal and ventral hippocampal poles remain unclear, we segmented the whole hippocampus into anterior (Bregma -1.06 to $-2.46 \mathrm{~mm}$ ) (incorporating dorsal) and posterior (Bregma -2.54 to $3.16 \mathrm{~mm}$ ) (incorporating ventral regions) levels.

FA values in the anterior and posterior hippocampus were significantly increased (Additional file 1: Figure 

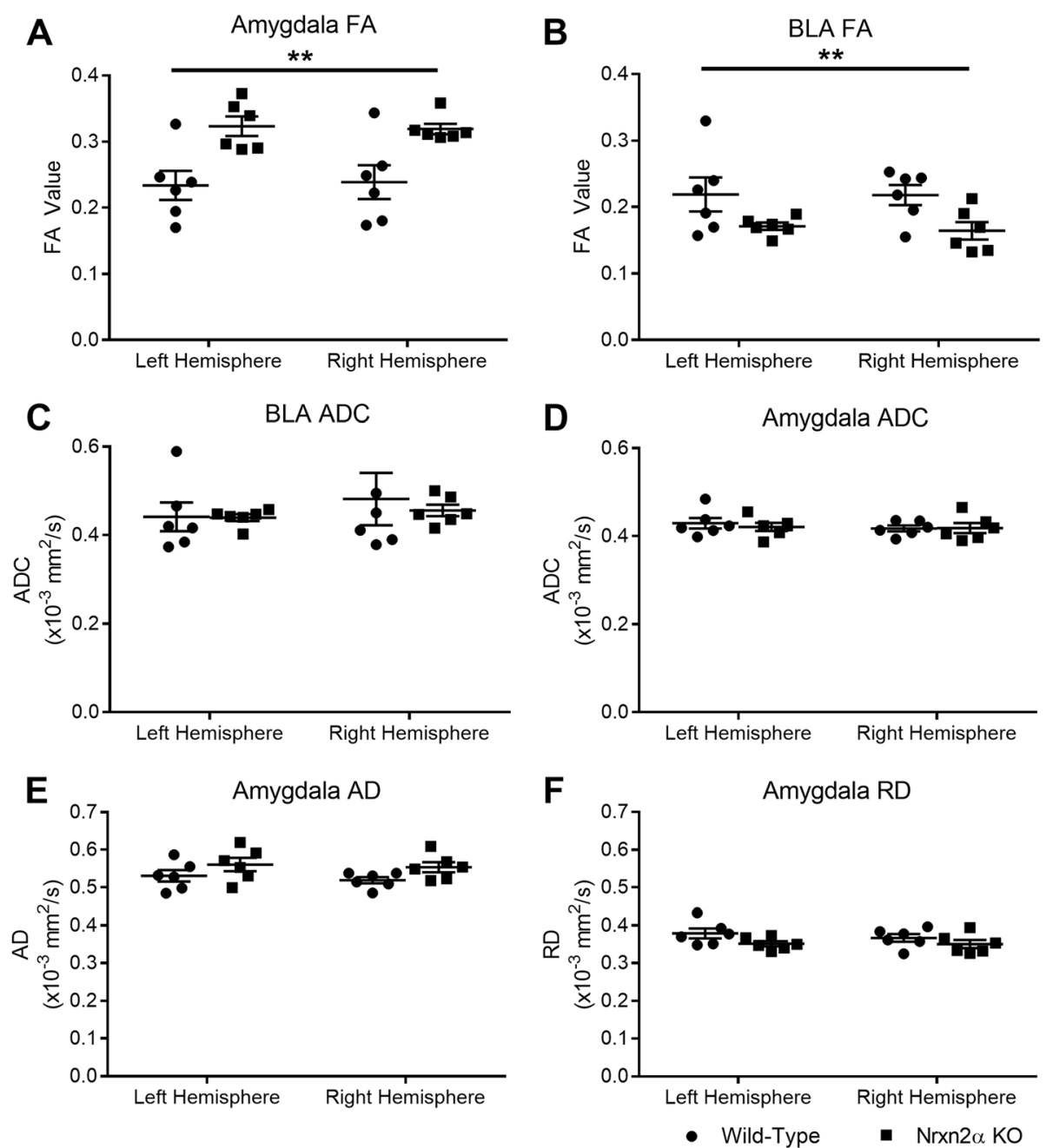

Fig. 2 Deletion of Nrxn2a increases amygdala fractional anisotropy (FA) but not apparent diffusion coefficient (ADC). DTI images of the amygdala was segmented at two regions; the whole amygdala in the anterior to posterior extent or the basolateral amygdala (BLA) centred at bregma $1.94 \mathrm{~mm}$. FA of the whole amygdala structure was significantly increased (a) but was decreased in the BLA (b). However, ADC was similar between the genotypes (c and $\mathbf{d}$ ). Axial (AD) (e) and radial diffusivity (RD) (f) was unaltered in the amygdala. ${ }^{* *} p<0.01,{ }^{*} p<0.05$. Error bars represent s.e.m. Wild type $n=6, \operatorname{Nr} \times n 2 a \mathrm{KO} n=6$

S4A and E; see figure legend for statistics). However, $\mathrm{ADC}$ was unaltered for the anterior and posterior hippocampus (Additional file 1: Figure S4B and F). AD was significantly increased in both the anterior and posterior hippocampal regions (Additional file 1: Figure S4C and G). RD was also significantly decreased in the anterior and posterior hippocampus in $N r x n 2 \alpha$ KO mice (Additional file 1: Figure S4D and $\mathrm{H}$ ).

Lastly, given DTI is most commonly associated with analysis of white matter tracts, we also quantified the corpus callosum. Changes within the corpus callosum have repeatedly been highlighted in autism $[55,56]$, including mouse models of autism [57, 58]. Here, we found significantly increased FA and reduced ADC in $N r x n 2 \alpha$ KO mice, which were driven by a significant reduction in RD (Additional file 1: Figure S6).
In summary, the microstructural measures most altered by $N r \times n 2 \alpha$ deletion were increases in FA, AD and $\mathrm{RD}$, including in the hippocampus, in line with recent work suggesting a role for ventral hippocampus in social memory [43].

\section{DTI tractography reveals Nrxn2a deletion affects} structural connectivity between the amygdala and orbitofrontal cortex

The amygdala is strongly and bidirectionally connected to both the hippocampus [59] and the OFC [60]. As all three regions are themselves important for social behaviour, and autism is thought to be, at least in part, related to abnormal structural connectivity [24], we performed tractography analysis between the amygdala (and 

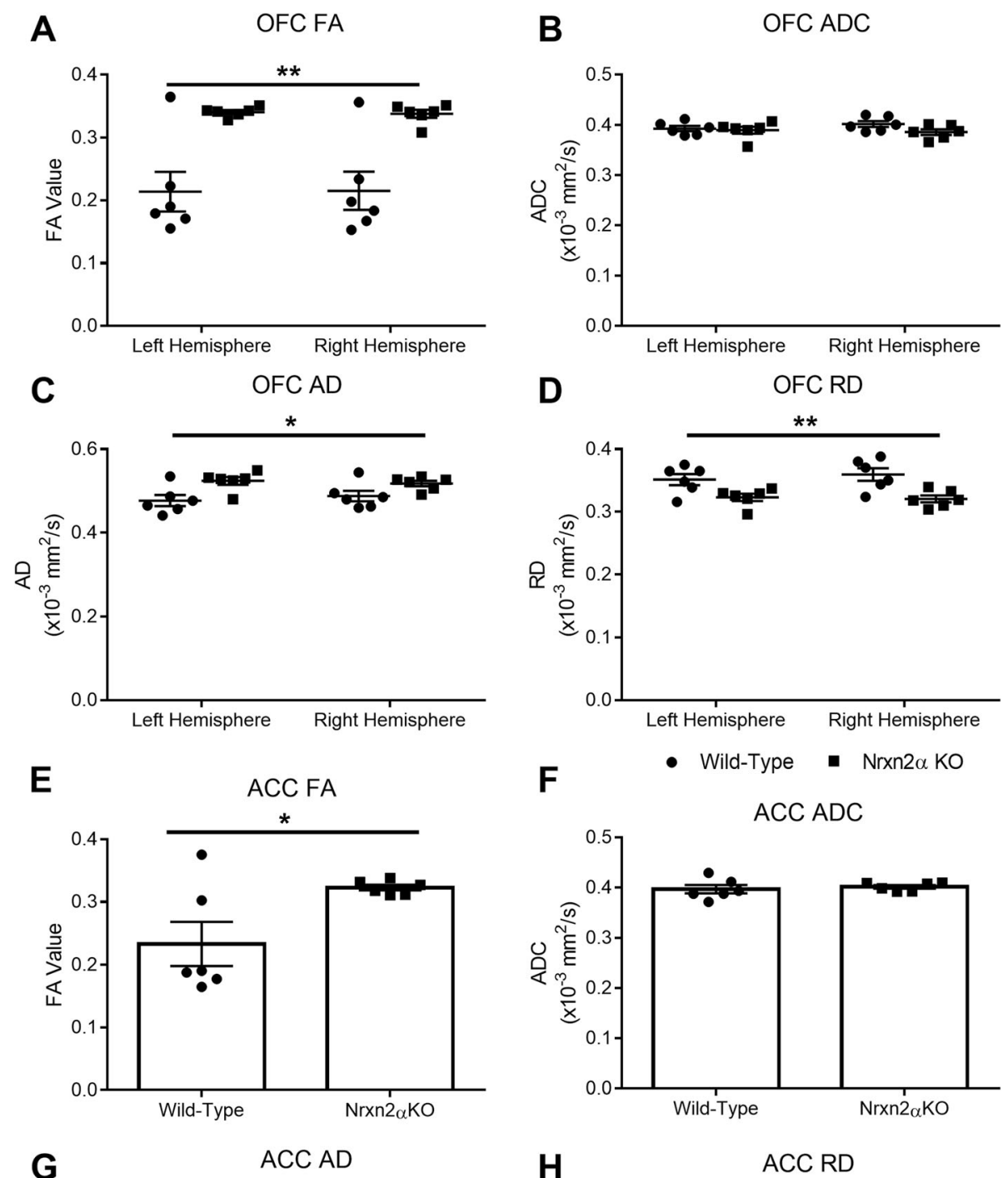

$\mathbf{F}$

- Wild-Type - Nrxn2 $\alpha$ KO

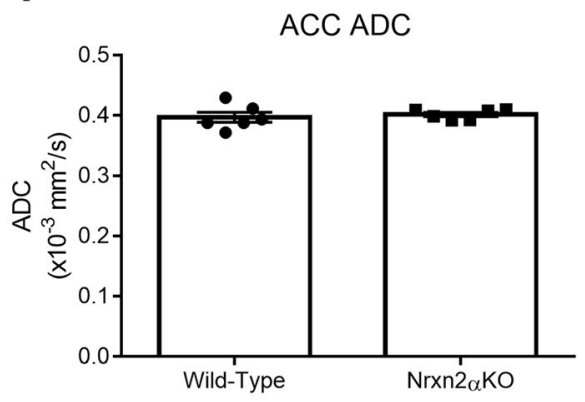

H

ACC RD
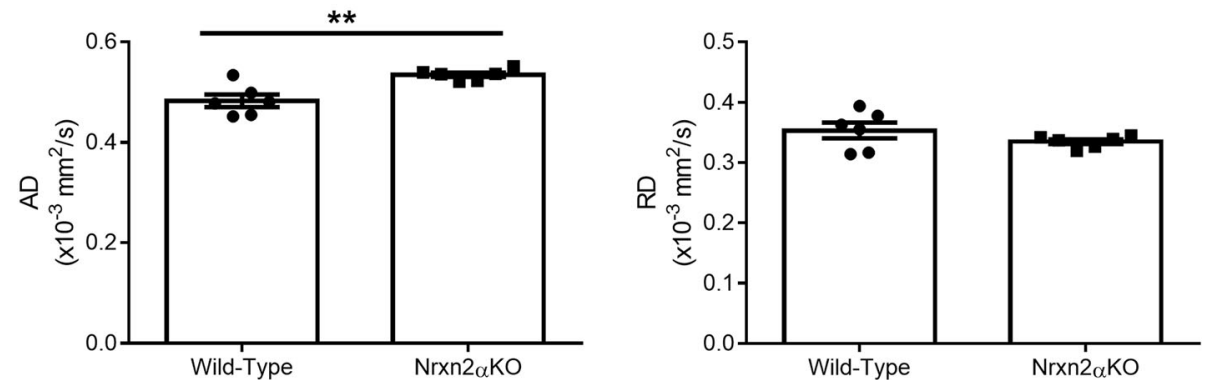

Fig. 3 Nrxnza KO mice have increased fractional anisotropy (FA) and axial (AD) and radial diffusivity (RD) in the orbitofrontal cortex (OFC) and the anterior cingulate cortex (ACC). FA was significantly different between wild-type and Nrxnza KO mice for FA in the OFC (a) and ACC (e), but ADC was not significantly changed in Nrxn2a KO mice in both prefrontal regions (b and $\mathbf{f}$ ). The OFC has significantly increased AD and RD (c and $\mathbf{d}$ ), whereas only AD was increased in the ACC $(\mathbf{g}-\mathbf{h}) .{ }^{* *} p<0.01,{ }^{*} p<0.05$. Error bars represent s.e.m. Wild type $n=6$, Nrxn2a KO $n=6$

specifically the BLA) and the hippocampus, and between the amygdala and the OFC.

From the anterior amygdala, we examined the diffusivity ( $\mathrm{AD}$ and $\mathrm{RD}$ ) of connections to the anterior and posterior hippocampus (Additional file 1: Figure S6). We did not observe differences in $\mathrm{RD}$ in the tracts connecting the amygdala with the hippocampus (see Additional file 1: Table S1 for non-significant statistics). Although AD between the anterior amygdala and anterior hippocampus did not differ by genotype, there was a 


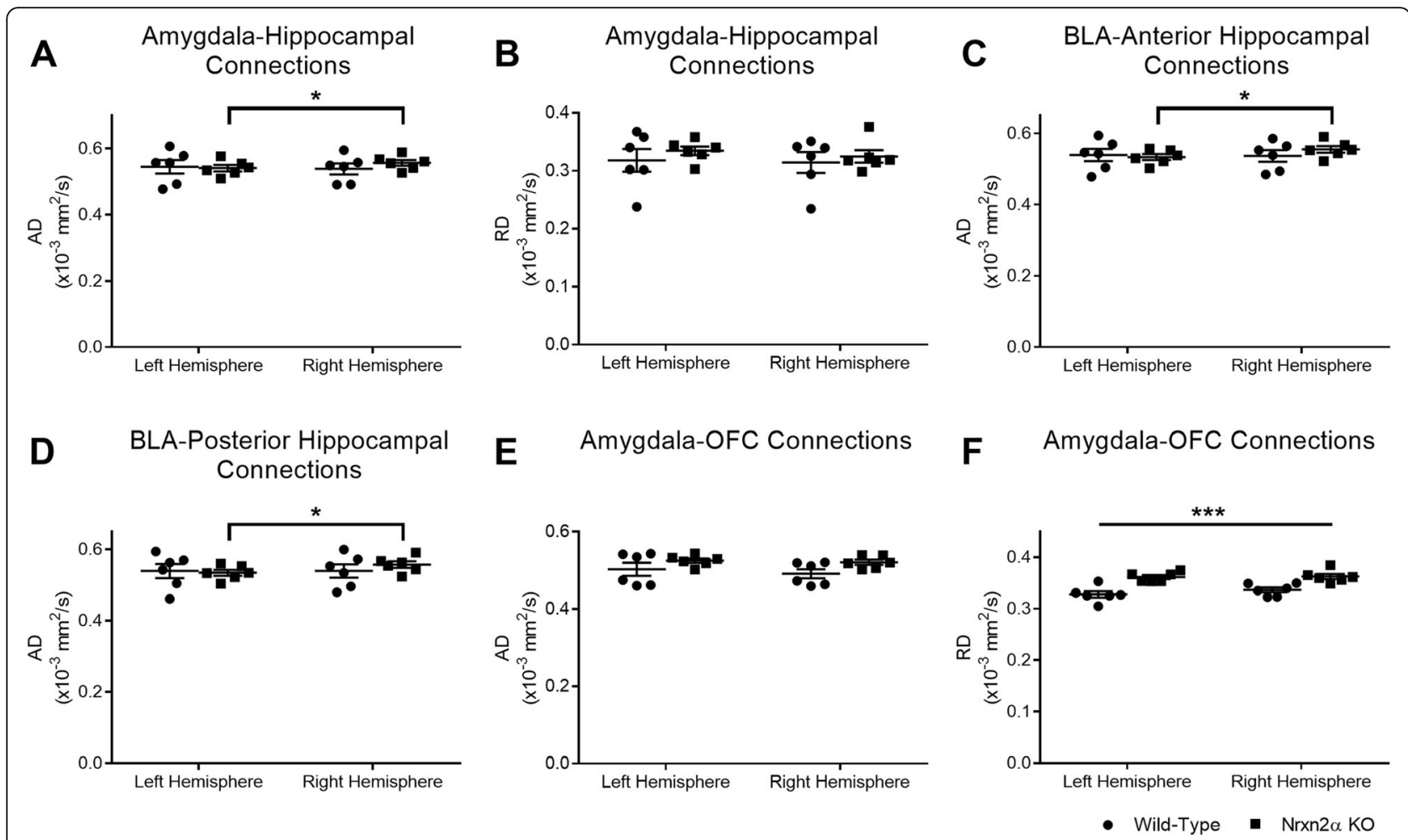

Fig. 4 Tractographic analysis of amygdala-hippocampus and amygdala-orbitofrontal cortex (OFC) connectivity. Amygdala-hippocampal connections are characterised by greater right hemisphere axial diffusivity (AD) in Nrxn2a KO mice (a) but not radial diffusivity (RD) (b). Specific to the BLA, connections to the anterior hippocampus (c) and posterior hippocampus (d) have greater right hemisphere AD. Although the amygdalaOFC connection was similar between the genotypes for AD (e), Nrxn2a KO mice had significantly increased RD (f). ${ }^{*} p<0.05$, ${ }^{* * *} p<0.001$. Error bars represent s.e.m. Wild type $n=6, \operatorname{Nrxn2a~KO} n=6$

significant interaction between the genotype and hemisphere (genotype $\times$ hemisphere $\left(F_{(1,10)}=12.12, p=\right.$ 0.023 , power $=88.0 \%$; Fig. $4 \mathrm{a}$ ); post hoc analysis shows this was driven by larger right-vs-left hemisphere $\mathrm{AD}$ values within the $\operatorname{Nrxn} 2 \alpha$ KOs only $(p=0.012)$. This difference could be driven by the BLA; there was increased $\mathrm{AD}$ in both the BLA/anterior hippocampus tracts (genotype $\times$ hemisphere $\left(F_{(1,10)}=10.53, p=0.032\right.$, power $=$ 83.2\%) and the BLA/posterior hippocampus tracts (genotype $\times$ hemisphere $\left(F_{(1,10)}=12.97, p=0.020\right.$, power $=90 \%$ ), which again was related to larger right-vs-left hemisphere values in the $N r x n 2 \alpha \mathrm{KOs}$ (BLA/anterior hippocampus $p=0.004$ and BLA/posterior hippocampus $p=0.001$, (Fig. $4 \mathrm{c}-\mathrm{d}$ )) but not the wild type (anterior $p=0.87$; posterior $p=1.00$ ). These results indicate that there are differences for the structural connectivity of the amygdala with the hippocampus within the left and right hemisphere in Nrxn $2 \alpha$ KO mice, with increased axial diffusivity in the right hemisphere. This finding is particularly interesting, as hemispheric differences in functional connectivity, particularly affecting connections from the right amygdala, have been found in children with ASD $[61,62]$.
Finally, we tested connections between the amygdala and the OFC. For AD, wild-type and Nrxn2 $\alpha \mathrm{KO}$ mice did not differ by genotype (Fig. 4e; genotype $\left(F_{(1,10)}=\right.$ $2.85, p=0.09)$, hemisphere $\left(F_{(1,10)}=6.38, p=0.052\right)$. RD was strikingly higher in Nrxn2 $\alpha \mathrm{KO}$ mice (Fig. 4f; genotype $\left(F_{(1,10)}=26.06, p=0.023\right.$, power $\left.\left.=99.5 \%\right)\right)$, indicative of a change in demyelination, axonal density or orientation [54].

\section{CLARITY reveals fibre disruption in Nrxn2a KO mice in the amygdala, orbitofrontal cortex and anterior cingulate cortex}

To further explore the differences as revealed by DTI, we performed CLARITY on the same brain tissue used in DTI and stained with neurofilament and DAPI to label axons and cell bodies, respectively. We were then able to derive both the axonal alignment (as in, the geometric alignment of axons (from linear alignment to random) within 3D space (see Additional file 1: Figure S2)) and density of the stained fibres, in addition to the cell density.

The pattern of results was broadly similar for both the prefrontal cortical ROIs. That is, first, axonal alignment was increased in $N r x n 2 \alpha$ KO mice in the ACC (Fig. $5 \mathrm{~d}$; 
A

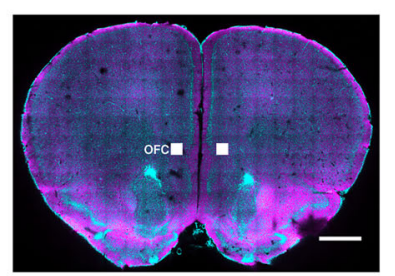

D ACC Axonal Alignment

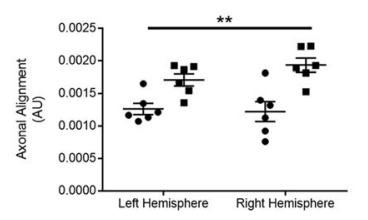

G Medial OFC
Axonal Alignment

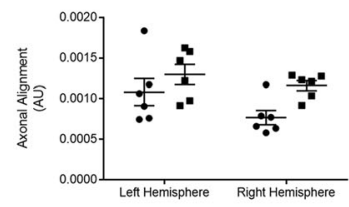

J BMA Axonal Alignment

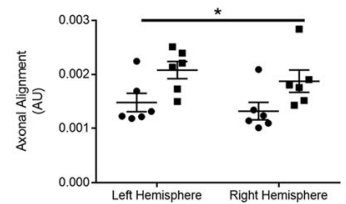

B

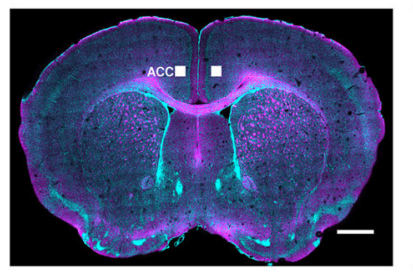

E

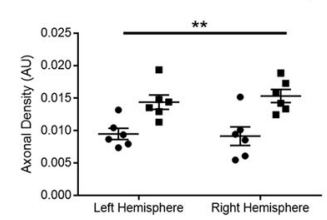

H
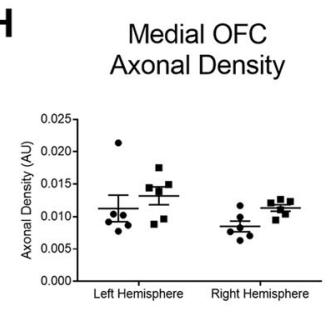

K BMA Axonal Density

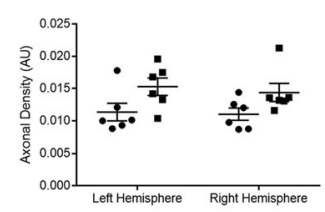

C

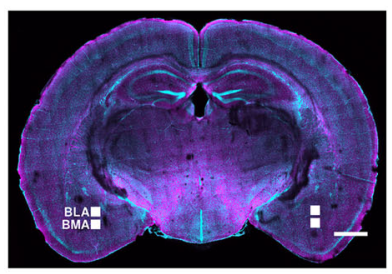

F

ACC

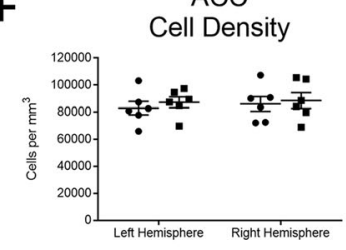

I

Medial OFC

Cell Density

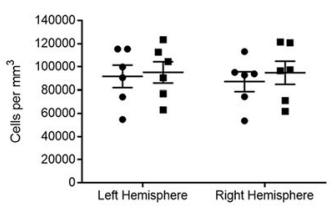

$\mathbf{L}$

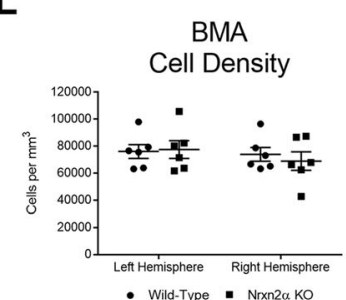

Fig. 5 CLARITY reveals differences in axonal alignment and fibre density in Nrxn2a KO mice. a-c Representative images of the CLARITY-treated brain, with ROI defined for the anterior cingulate cortex (ACC), orbitofrontal cortex (OFC), basomedial amygdala (BMA) and basolateral amygdala (BLA). For the ACC, the axonal alignment (d) and axon density (e) were significantly altered in KO mice, but cell density was unaltered (f). Within the medial OFC, only axonal alignment was significantly altered in $\mathrm{KOs}(\mathbf{g})$, with axon density (h) and cell density (i) being similar. For the BMA, both the axonal alignment (j) and axon density (k) were significantly increased, while cell density was unaltered (I). ${ }^{*} p<0.05,{ }^{* *} p<0.01$. Error bars represent s.e.m. Wild type $n=6, \operatorname{Nrxn2a} \mathrm{KO} n=6$

genotype $\left(F_{(1,10)}=16.06, p=0.011\right.$, power $\left.=94.9 \%\right)$ but not the OFC (Fig. 5g; genotype $\left(F_{(1,10)}=5.56, p=\right.$ 0.059). Second, this could not be explained by a difference in cell density, since that was similar between the $\mathrm{KO}$ and wild-type mice in both the ACC (Fig. 5f; genotype $\left(F_{(1,10)}<1\right)$, hemisphere $\left(F_{(1,10)}=1.73, p=0.11\right)$ and the OFC (Fig. 5h; genotype $\left(F_{(1,10)}=3.09, p=0.08\right.$ ). An increase in axonal density in Nrxn $2 \alpha \mathrm{KO}$ mice was reliable in the ACC (Fig. 5e; genotype $\left(F_{(1,10)}=14.64\right.$, $p=0.014$, power $=93.0 \%$ ), but not in the OFC (Fig. 5h; genotype $\left(F_{(1,10)}=3.09, p=0.083\right)$.

We further examined two regions of the anterior amygdala, the BLA and basomedial (BMA) nuclei, where altered social cellular responses have been reported in human autism [38]. We did not observe any significant differences for axonal alignment or fibre density in the BLA (see Additional file 1: Figure S7A-C), whereas axonal alignment (Fig. 5j; genotype $F_{(1,10)}=7.70, p=$ 0.045 , power $=70.6 \%$ ) but not axonal density (Fig. 5k; genotype $\left(F_{(1,10)}=6.10, p=0.054\right)$ was increased in $N r \times n 2 \alpha$ KO mice in the basomedial nuclei, while cell density was unaffected (Fig. 5l; genotype $\left(F_{(1,10)}<1\right)$. Alterations in axonal alignment and density as directly revealed by CLARITY could explain the increases in diffusivity and RD in the prefrontal regions, as measured by DTI.

To test the specificity of these alterations, we examined three further brain regions: the primary motor cortex (M1; Additional file 1: Figure S7D-F); the primary somatosensory cortex (S1; Additional file 1: Figure S7H- 
$\mathrm{J}$ ); and the barrel field (BF; Additional file 1: Figure S7K-M). Interestingly, although there were differences between the hemispheres, there were no statistical differences between the genotypes or genotype $\times$ hemisphere interactions for any measure (Additional file 1: Table S2), suggesting some specificity of the alterations in social-relevant brain regions in Nrxn2 $\alpha \mathrm{KO}$ mice.

In summary, in both the prefrontal ROIs, namely the OFC and the ACC, DTI showed that ADC and RD were increased in $N r x n 2 \alpha$ KO mice, likely related to complementary analysis from CLARITY showing that axonal alignment was altered in $N r x n 2 \alpha \mathrm{KO}$ mice in both prefrontal ROIs.

\section{Discussion}

Interestingly, the single-gene deletion of $N r \times n 2 \alpha$ captures several key aspects of human ASD. In terms of behaviour, three studies have now found social deficits associated with $N r x n 2 \alpha \mathrm{KO}$ [17-19]; in terms of brain structure, as reported here (summarised below), the Nrxn $2 \alpha$ KO mouse model shows altered microstructure and structural connectivity patterns in socially relevant brain regions reminiscent of changes in ASD.

A DTI approach has been used for some time to explore neuropathological markers in autistic patients; alterations in myelination, axonal abundance, size and orientation all modify FA and ADC values [21, 63], specifically by reducing amygdala FA $[23,63]$, and have been used as a quantitative measure of changes to brain white matter integrity [23, 24]. However, several studies have noted increases in FA in ASD patients (see Table 1 of [64]). Furthermore, both increased RD of various white matter tracts $[65,66]$ and increased whole-brain AD [66] have been observed in ASD. The Nrxn2a KO mouse reproduces some of these specific changes, including altered FA and increases in $\mathrm{ADC}, \mathrm{AD}$ and $\mathrm{RD}$. Whole brain increases in ADC, AD and RD (but not FA) have been reported in ASD children, as have increases in $\mathrm{ADC}$ and RD in frontal cortex tracts [66]. FA has been noted as reduced in the amygdala in ASD children and adolescents [67], and right-sided lateralisation of abnormal amygdala/hippocampus-related connections, as seen in our Nrxn2a KO mouse, has been noted in high-functioning adolescents/adults with autism [68].

While the current study specifically explores structural connectivity, it is difficult to extrapolate as to what these structural changes mean for functional connectivity in the $N r x n 2 \alpha$ KO mouse. Hyper and hypo connectivity theories of autism have remained contentious and vary in humans by cohort studied (e.g. by age of participant) [69]. Further, in studies that have combined resting-state functional MRI (rsfMRI) and DTI, functional and structural connectivity do not always overlap [70-72]. Our current data suggests that DTI differences can be explained by altered axonal patterning (i.e. CLARITY). Others have explored the biological mechanisms linking structural connectivity to altered functional connectivity. Zhan et al. found that deletion of the chemokine receptor Cx3cr1 resulted in impaired synaptic pruning of long-range connections during development, which manifested as impaired social behaviour caused by decreased frontal functional connectivity, reduced synaptic multiplicity and weakened coherence of local field potentials [73]. Thus, it is possible that impairments in neuronal structural maturation can generate functional connectivity deficits that encapsulate core autism phenotypes.

Our findings corroborate these quantifications of clinical autism but highlight the question of what do the different measures of ADC, FA, AD and RD represent? Importantly, we observed these microstructural changes in various socially relevant brain regions against a background of unchanged cell density in all our study's ROIs. Unexpectedly, this highlights the power of our new approach. Dudanova et al. concluded from measures of cell counting and cortical cell layer thickness that NRXN2 played little role in normal brain development [20]. Indeed, in earlier studies, it was suggested that deletion of all Nrxns was unlikely to affect synaptic development but instead disrupts synaptic function [74]. We propose that measures such as two-dimensional cell counting may be underestimating the impact of genetic mutations upon normal development. By staining cleared brain tissue with a nuclear marker and performing automated three-dimensional cell counting, we found no effect of $N r \times n 2 \alpha$ deletion on cell density in any region of interest examined. But this belies the clear effects upon microstructure integrity across multiple regions as measured by both DTI and CLARITY and its specificity; only the socially relevant brain regions we tested were disrupted, and not primary sensory or motor regions. Future studies will benefit from employing more sensitive measures of brain structural connectivity to determine the relevance of genetic mutations in development.

FA and ADC can be influenced by changes in axonal density and alignment (e.g. by myelination, demyelination, axonal damage, loss of white matter coherence [75]). It is likely that the axonal alignment metric used to quantify CLARITY more closely reflects the ADC measure of DTI, given that ADC (or mean diffusivity) equally weighs diffusion across all eigenvectors and does not bias the primary eigenvector as FA does. Thus, it is likely that alterations in the properties of axons in $N r x n 2 \alpha \mathrm{KO}$ mice are driving these changes in FA and ADC. Given we see differences in RD, thought to reflect tract branching and myelination (as it measures $\lambda_{2}$ and $\lambda_{3}$ ), it is possible that the orientation in the perpendicular not parallel orientation of fibres is mostly affected. 
Given the differences in the amygdala, OFC and ACC, it is possible that even though neuronal densities are similar in the Nrxn $2 \alpha \mathrm{KO}$ brain, it is the connections between neurones and brain regions that are perturbed. This would be consistent with the idea that structural connectivity disruption may represent a core feature of autism [76]. A broader question is how does the loss of $N r x n 2 \alpha$ account for changes in axonal organisation? Ultimately, this question requires further studies. Others have shown that in Nrxn2 $\alpha$ KO mice, excitatory transmitter release is reduced, as is short-term plasticity [18]. Reduced glutamatergic release, even at a relatively long range to the synapse, can change the complexity of dendritic arbours [77]. As this is a gene deletion model, it is conceivable that altered glutamatergic signalling during early development impairs appropriate synapse maturation, leading to the structural changes we see herein. Further, how or whether these structural changes fully explain the social impairments of $N r x n 2 \alpha \mathrm{KO}$ mice would require new studies. Conceivably, inducible knockdown of Nrxn2 (by inducible knockout, siRNA, optogenetics etc.) within a specific brain region would provide evidence that social abnormalities are being driven by Nrxn2 loss. However, developmentally dependent altered structural connectivity would be harder to definitively manipulate to explain changes in social behaviours.

Here we have developed a new application of CLARITY to quantitatively investigate disease models by combining DTI with high-resolution 3D imaging and automated analysis of axonal fibres in a within-subject study. Inevitably, there are some technical limitations that will require future refinement as this technology matures.

First, while we used CLARITY and immunolabelling to identify axons, we cannot know whether axon-related changes alone reflect all the changes we observed for our DTI measures. Second, while we can segment entire brain regions for DTI analysis, it was not practical to image larger brain areas at the necessary resolution for CLARITY. While it is theoretically possible that we may bias sampling of each brain region by picking ROIs for multiphoton imaging, this was done using atlas-defined coordinates and by an experimenter blind to the DTI results, so minimising any bias. However, within the current study, we were only able to apply the CLARITY approach to the amygdala, OFC and ACC. It was not practical to apply this methodology to the hippocampus, due to its extremely heterogeneous structure. The small cubic ROIs could not be reproducibly positioned, and larger ROIs to average across larger areas of the hippocampus were not possible. Although imaging of fibre tracts in large volumes of cleared tissue is possible [78], fluorescent labelling limitations make it impractical for a study of this nature. Despite this, as the adoption of the CLARITY technique increases, we hope that the use of DTI and CLARITY to study structural connectivity across spatial scales will become a commonplace.

As yet, no one DTI protocol has emerged as the standard for in vivo or ex vivo imaging. Indeed, there has been debate regarding the best number of diffusion gradients to use, among other parameters [79]. Undoubtedly, more directions that what we used here would facilitate better interpretations, this is a limitation of the current work. Despite this, the major purpose of the current paper is to develop a new generation of CLARITY analysis. We hope that future studies will refine on both DTI and CLARITY parameters to maximise analysis methodology. A further potential limitation of the current study is that groups of six animals may be underpowered. We argue for our approach here as follows. First, low variance in the datasets permits smaller group sizes. Second, for most of our significant results, the observed power was more than $80 \%$. Third, given the technical complexity of this approach, particularly in its early adoption and refinement stages, large sample throughput of multiple brain regions is challenging.

In summary, our combined use of DTI and CLARITY has revealed changes in microstructure and structural connectivity of socially relevant brain regions in $N r \times n 2 \alpha$ $\mathrm{KO}$ mice that may underlie their deficits in social behaviour. It is hard to conceive how these changes could have been observed using classical experimental approaches. We envisage this approach will deliver a new level of detail in structural connectivity approaches to understanding autism.

\section{Additional file}

\section{Additional file 1: Supplemental materials and methods}

(DOCX 6377 kb)

\section{Abbreviations}

ACC: Anterior cingulate cortex; AD: Axial diffusivity; ADC: Apparent diffusion coefficient; ASD: Autism spectrum disorder; BLA: Basolateral amygdala; CLARITY: Optically cleared brain tissue; DTI: Diffusion tensor imaging; FA: Fractional anisotropy; Nrxn2: Neurexin II; OFC: Orbitofrontal cortex; RD: Radial diffusivity; ROI: Region of interest

\section{Acknowledgements}

Not applicable.

\section{Funding}

This work was supported by the Guy's and St. Thomas' Charity Prize PhD scholarship to ALT, a Medical Research Council (UK) grant (G0900625) to SJC and RJR, a University of Leeds Wellcome Trust ISSF (UK) Fellowship, a Royal Society (UK) grant (RG130316), an Alzheimer's Society Fellowship (AS-JF-15008) to JD, a British Pharmacological Society (UK) grant to JD and CL, a BBSRC grant to LCA (BB/P000479/1) and a BBSRC grant to CL (BB/M008975/ 1). We acknowledge financial support from the Innovative Medicines Initiative Joint Undertaking under grant agreement no. 115300, resources of which are composed of financial contribution from the European Union's Seventh Framework Programme (FP7/2007-2013) and EFPIA companies' in 
kind contribution, the Mortimer D Sackler Foundation and the Sackler Institute for Translational Neurodevelopment (ALT and LCA). Some analysis scripts were provided to ALT at the Computational Image Analysis in Cellular and Developmental Biology course at the Marine Biological Laboratory (Woods Hole, MA, USA), funded by National Institutes of Health (R25 GM103792-01).

\section{Availability of data and materials}

The codes used to quantify the CLARITY datasets are made available by author LCA by email request to authors LCA or JD, subject to reference to the current paper. The datasets used and/or analysed during the current study are available from the corresponding author on reasonable request.

\section{Authors' contributions}

$E P, A L T, L C A$ and JD conceived the study. EP and ALT performed the experiments. EP, ALT, LCA and JD analysed the data. SJC, RJR, LCA and JD funded the study. All authors contributed to writing the paper. All authors have read and approved the final manuscript.

\section{Ethics approval and consent to participate}

All experiments were performed under UK Home Office Project and Personal Licenses in accordance with the Animals (Scientific Procedures) Act 1986, and with the approval of the University of Leeds and Durham University Animal Ethical and Welfare Review Boards.

\section{Consent for publication}

Not applicable

\section{Competing interests}

The authors declare that they have no competing interests.

\section{Publisher's Note}

Springer Nature remains neutral with regard to jurisdictional claims in published maps and institutional affiliations.

\section{Author details}

${ }^{1}$ School of Biomedical Sciences, University of Leeds, Leeds LS2 9JT, UK. ${ }^{2}$ Centre for Developmental Neurobiology, Institute of Psychiatry, Psychology and Neuroscience, King's College London, London SE1 1UL, UK. ${ }^{3}$ MRC Centre for Neurodevelopmental Disorders, King's College London, London SE1 1UL, UK. ${ }^{4}$ Department of Forensic and Neurodevelopmental Sciences, Institute of Psychiatry, Psychology and Neuroscience, King's College London, London SE5 8AF, UK. ${ }^{5}$ Department of Psychology, Durham University, South Road, Durham DH1 3LE, UK. ${ }^{6}$ Robarts Research Institute, Western University, London, ON N6A 5B7, Canada. ${ }^{7}$ School of Psychology, University of Leeds, Leeds LS2 9JT, UK.

\section{Received: 15 October 2018 Accepted: 18 February 2019} Published online: 28 February 2019

\section{References}

1. Sandin S, Lichtenstein P, Kuja-Halkola R, Larsson H, Hultman CM, Reichenberg A. The familial risk of autism. Jama. 2014;311(17):1770-7.

2. Cardno AG, Gottesman II. Twin studies of schizophrenia: from bow-andarrow concordances to star wars Mx and functional genomics. Am J Med Genet. 2000;97(1):12-7.

3. Ronald A, Happe F, Bolton P, Butcher LM, Price TS, Wheelwright S, et al. Genetic heterogeneity between the three components of the autism spectrum: a twin study. J Am Acad Child Adolesc Psychiatry. 2006;45(6):691-9.

4. Ronemus M, lossifov I, Levy D, Wigler M. The role of de novo mutations in the genetics of autism spectrum disorders. Nat Rev Genet. 2014;15(2):133-41.

5. Geschwind DH, State MW. Gene hunting in autism spectrum disorder: on the path to precision medicine. Lancet Neurol. 2015;14(11):1109-20.

6. Sahin M, Sur M. Genes, circuits, and precision therapies for autism and related neurodevelopmental disorders. Science. 2015;350(6263). https://doi. org/10.1126/science.aab3897.

7. Lise MF, El-Husseini A. The neuroligin and neurexin families: from structure to function at the synapse. Cell Mol Life Sci. 2006;63(16):1833-49.

8. Sudhof TC. Synaptic neurexin complexes: a molecular code for the logic of neural circuits. Cell. 2017;171(4):745-69.
9. Gauthier J, Siddiqui TJ, Huashan P, Yokomaku D, Hamdan FF, Champagne N, et al. Truncating mutations in NRXN2 and NRXN1 in autism spectrum disorders and schizophrenia. Hum Genet. 2011;130(4):563-73.

10. Mohrmann I, Gillessen-Kaesbach G, Siebert R, Caliebe A, Hellenbroich Y. A de novo $0.57 \mathrm{Mb}$ microdeletion in chromosome 11q13.1 in a patient with speech problems, autistic traits, dysmorphic features and multiple endocrine neoplasia type 1. Eur J Med Genet. 2011;54(4):e461-4.

11. Boyle MI, Jespersgaard C, Nazaryan L, Ravn K, Brondum-Nielsen K, Bisgaard AM, et al. Deletion of 11q12.3-11q13.1 in a patient with intellectual disability and childhood facial features resembling Cornelia de Lange syndrome. Gene. 2015;572(1):130-4

12. Li J, Wang L, Gou H, Shi L, Zhang K, Tang M, et al. Targeted sequencing and functional analysis reveal brain-size-related genes and their networks in autism spectrum disorders. Mol Psychiatry. 2017;22:1282-90.

13. Yuan H, Li X, Wang Q, Yang W, Song J, Hu X, et al. A de novo $921 \mathrm{~Kb}$ microdeletion at 11q13.1 including neurexin 2 in a boy with developmental delay, deficits in speech and language without autistic behaviors. Eur J Med Genet. 2018;61(10):607-11.

14. Williams SM, An JY, Edson J, Watts M, Murigneux V, Whitehouse AJO, et al. An integrative analysis of non-coding regulatory DNA variations associated with autism spectrum disorder. Mol Psychiatry. 2018. https://doi.org/10. 1038/s41380-018-0049-X.

15. Wang J, Gong J, Li L, Chen Y, Liu L, Gu H, et al. Neurexin gene family variants as risk factors for autism spectrum disorder. Autism Res. 2018;11(1):37-43.

16. Duda M, Zhang H, Li HD, Wall DP, Burmeister M, Guan Y. Brain-specific functional relationship networks inform autism spectrum disorder gene prediction. Transl Psychiatry. 2018;8(1):56.

17. Dachtler J, Glasper J, Cohen RN, Ivorra JL, Swiffen DJ, Jackson AJ, et al. Deletion of alpha-neurexin II results in autism-related behaviors in mice. Transl Psychiatry. 2014;4:e484.

18. Born G, Grayton HM, Langhorst H, Dudanova I, Rohlmann A, Woodward BW et al. Genetic targeting of NRXN2 in mice unveils role in excitatory cortical synapse function and social behaviors. Front Synaptic Neurosci. 2015;7:3.

19. Dachtler J, Ivorra JL, Rowland TE, Lever C, Rodgers RJ, Clapcote SJ. Heterozygous deletion of alpha-neurexin I or alpha-neurexin II results in behaviors relevant to autism and schizophrenia. Behav Neurosci. 2015;129(6):765-76.

20. Dudanova I, Tabuchi K, Rohlmann A, Sudhof TC, Missler M. Deletion of alpha-neurexins does not cause a major impairment of axonal pathfinding or synapse formation. J Comp Neurol. 2007;502(2):261-74.

21. Beaulieu $C$. The basis of anisotropic water diffusion in the nervous system a technical review. NMR Biomed. 2002;15(7-8):435-55.

22. Barnea-Goraly N, Kwon H, Menon V, Eliez S, Lotspeich L, Reiss AL. White matter structure in autism: preliminary evidence from diffusion tensor imaging. Biol Psychiatry. 2004;55(3):323-6.

23. Noriuchi M, Kikuchi Y, Yoshiura T, Kira R, Shigeto H, Hara T, et al. Altered white matter fractional anisotropy and social impairment in children with autism spectrum disorder. Brain Res. 2010;1362:141-9.

24. Belmonte MK, Allen G, Beckel-Mitchener A, Boulanger LM, Carper RA, Webb SJ. Autism and abnormal development of brain connectivity. J Neurosci. 2004;24(42):9228-31.

25. Chung K, Wallace J, Kim SY, Kalyanasundaram S, Andalman AS, Davidson TJ, et al. Structural and molecular interrogation of intact biological systems. Nature. 2013;497(7449):332-7.

26. Pervolaraki E, Anderson RA, Benson AP, Hayes-Gill B, Holden AV, Moore BJ, et al. Antenatal architecture and activity of the human heart. Interface focus. 2013;3(2):20120065.

27. Pervolaraki E, Dachtler J, Anderson RA, Holden AV. Ventricular myocardium development and the role of connexins in the human fetal heart. Sci Rep. 2017;7(1):12272

28. Yeh FC, Verstynen TD, Wang Y, Fernandez-Miranda JC, Tseng WY. Deterministic diffusion fiber tracking improved by quantitative anisotropy. PLoS One. 2013;8(11):e80713.

29. Paxinos G, Franklin K. The mouse brain in stereotaxic coordinates. 3rd ed. London: Academic Press; 2008.

30. Basser PJ, Mattiello J, LeBihan D. Estimation of the effective self-diffusion tensor from the NMR spin echo. J Magn Reson Series B. 1994;103(3):247-54.

31. Jiang H, van Zijl PC, Kim J, Pearlson GD, Mori S. DtiStudio: resource program for diffusion tensor computation and fiber bundle tracking. Comput Methods Prog Biomed. 2006;81(2):106-16.

32. Ferguson JN, Aldag JM, Insel TR, Young LJ. Oxytocin in the medial amygdala is essential for social recognition in the mouse. J Neurosci. 2001;21(20):8278-85. 
33. Tanimizu T, Kenney JW, Okano E, Kadoma K, Frankland PW, Kida S. Functional connectivity of multiple brain regions required for the consolidation of social recognition memory. J Neurosci. 2017;37(15):4103-16.

34. Emery NJ, Capitanio JP, Mason WA, Machado CJ, Mendoza SP, Amaral DG. The effects of bilateral lesions of the amygdala on dyadic social interactions in rhesus monkeys (Macaca mulatta). Behav Neurosci. 2001;115(3):515-44.

35. Rosvold HE, Mirsky AF, Pribram KH. Influence of amygdalectomy on social behavior in monkeys. J Comp Physiol Psychol. 1954;47(3):173-8.

36. Mosher CP, Zimmerman PE, Gothard KM. Neurons in the monkey amygdala detect eye contact during naturalistic social interactions. Curr Biol. 2014; 24(20):2459-64.

37. Rutishauser U, Mamelak AN, Adolphs R. The primate amygdala in social perception - insights from electrophysiological recordings and stimulation. Trends Neurosci. 2015;38(5):295-306.

38. Rutishauser U, Tudusciuc O, Wang S, Mamelak AN, Ross IB, Adolphs R. Single-neuron correlates of atypical face processing in autism. Neuron. 2013; 80(4):887-99.

39. Adolphs $R$, Tranel $D$, Damasio AR. The human amygdala in social judgment. Nature. 1998;393(6684):470-4.

40. Baron-Cohen S, Ring HA, Bullmore ET, Wheelwright S, Ashwin C, Williams SC. The amygdala theory of autism. Neurosci Biobehav Rev. 2000;24(3):355-64.

41. Maaswinkel H, Baars AM, Gispen WH, Spruijt BM. Roles of the basolateral amygdala and hippocampus in social recognition in rats. Physiol Behav. 1996;60(1):55-63.

42. Kogan JH, Frankland PW, Silva AJ. Long-term memory underlying hippocampus-dependent social recognition in mice. Hippocampus. 2000; 10(1):47-56

43. Okuyama T, Kitamura T, Roy DS, Itohara S, Tonegawa S. Ventral CA1 neurons store social memory. Science. 2016;353(6307):1536-41.

44. van der Kooij MA, Fantin M, Kraev I, Korshunova I, Grosse J, Zanoletti O, et al. Impaired hippocampal neuroligin-2 function by chronic stress or synthetic peptide treatment is linked to social deficits and increased aggression. Neuropsychopharmacology. 2014;39(5):1148-58.

45. Rubin RD, Watson PD, Duff MC, Cohen NJ. The role of the hippocampus in flexible cognition and social behavior. Front Hum Neurosci. 2014;8:742.

46. Nicolson R, DeVito TJ, Vidal CN, Sui Y, Hayashi KM, Drost DJ, et al. Detection and mapping of hippocampal abnormalities in autism. Psychiatry Res. 2006; 148(1):11-21.

47. Schumann CM, Hamstra J, Goodlin-Jones BL, Lotspeich LJ, Kwon H, Buonocore $\mathrm{MH}$, et al. The amygdala is enlarged in children but not adolescents with autism; the hippocampus is enlarged at all ages. J Neurosci. 2004;24(28):6392-401.

48. Bachevalier J, Loveland KA. The orbitofrontal-amygdala circuit and selfregulation of social-emotional behavior in autism. Neurosci Biobehav Rev. 2006;30(1):97-117.

49. Beer JS, John OP, Scabini D, Knight RT. Orbitofrontal cortex and social behavior: integrating self-monitoring and emotion-cognition interactions. J Cogn Neurosci. 2006;18(6):871-9.

50. Watson KK, Platt ML. Social signals in primate orbitofrontal cortex. Curr Biol. 2012;22(23):2268-73.

51. Girgis RR, Minshew NJ, Melhem NM, Nutche JJ, Keshavan MS, Hardan AY. Volumetric alterations of the orbitofrontal cortex in autism. Prog NeuroPsychopharmacol Biol Psychiatry. 2007;31(1):41-5.

52. Tyson AL, Hilton ST, Andreae LC. Rapid, simple and inexpensive production of custom 3D printed equipment for large-volume fluorescence microscopy. Int J Pharm. 2015;494(2):651-6.

53. Herbert MR, Ziegler DA, Deutsch CK, O'Brien LM, Kennedy DN, Filipek PA, et al. Brain asymmetries in autism and developmental language disorder: a nested whole-brain analysis. Brain. 2005;128(Pt 1):213-26.

54. Alexander AL, Lee JE, Lazar M, Field AS. Diffusion tensor imaging of the brain. Neurotherapeutics. 2007:4(3):316-29.

55. Alexander AL, Lee JE, Lazar M, Boudos R, DuBray MB, Oakes TR, et al. Diffusion tensor imaging of the corpus callosum in autism. Neurolmage. 2007:34(1):61-73.

56. Travers BG, do PM T, Adluru N, Lange N, Destiche D, Ennis C, et al. Atypical development of white matter microstructure of the corpus callosum in males with autism: a longitudinal investigation. Mol Autism. 2015;6:15.

57. Ellegood J, Babineau BA, Henkelman RM, Lerch JP, Crawley JN. Neuroanatomical analysis of the BTBR mouse model of autism using magnetic resonance imaging and diffusion tensor imaging. Neurolmage. 2013;70:288-300.
58. Kumar M, Kim S, Pickup S, Chen R, Fairless AH, Ittyerah R, et al. Longitudinal in-vivo diffusion tensor imaging for assessing brain developmental changes in BALB/CJ mice, a model of reduced sociability relevant to autism. Brain Res. 2012;1455:56-67.

59. Pitkanen A, Pikkarainen M, Nurminen N, Ylinen A. Reciprocal connections between the amygdala and the hippocampal formation, perirhinal cortex, and postrhinal cortex in rat. A review. Ann N Y Acad Sci. 2000;911:369-91.

60. Cavada C, Company T, Tejedor J, Cruz-Rizzolo RJ, Reinoso-Suarez F. The anatomical connections of the macaque monkey orbitofrontal cortex. A review. Cereb Cortex. 2000;10(3):220-42.

61. Abrams DA, Lynch CJ, Cheng KM, Phillips J, Supekar K, Ryali S, et al. Underconnectivity between voice-selective cortex and reward circuitry in children with autism. Proc Natl Acad Sci U S A. 2013;110(29):12060-5.

62. Murphy ER, Foss-Feig J, Kenworthy L, Gaillard WD, Vaidya CJ. Atypical functional connectivity of the amygdala in childhood autism spectrum disorders during spontaneous attention to eye-gaze. Autism Res Treat. 2012; 2012:652408.

63. Sundaram SK, Kumar A, Makki MI, Behen ME, Chugani HT, Chugani DC. Diffusion tensor imaging of frontal lobe in autism spectrum disorder. Cereb Cortex. 2008;18(11):2659-65.

64. Travers BG, Adluru N, Ennis C, do PM T, Destiche D, Doran S, et al. Diffusion tensor imaging in autism spectrum disorder: a review. Autism Res. 2012;5(5):289-313.

65. Ameis SH, Fan J, Rockel C, Voineskos AN, Lobaugh NJ, Soorya L, et al. Impaired structural connectivity of socio-emotional circuits in autism spectrum disorders: a diffusion tensor imaging study. PLoS One. 2011;6(11):e28044.

66. Shukla DK, Keehn B, Muller RA. Tract-specific analyses of diffusion tensor imaging show widespread white matter compromise in autism spectrum disorder. J Child Psychol Psychiatry. 2011;52(3):286-95.

67. Jou RJ, Jackowski AP, Papademetris X, Rajeevan N, Staib LH, Volkmar FR. Diffusion tensor imaging in autism spectrum disorders: preliminary evidence of abnormal neural connectivity. Aust N Z J Psychiatry. 2011;45(2):153-62.

68. Conturo TE, Williams DL, Smith CD, Gultepe E, Akbudak E, Minshew NJ. Neuronal fiber pathway abnormalities in autism: an initial MRI diffusion tensor tracking study of hippocampo-fusiform and amygdalo-fusiform pathways. J Int Neuropsychol Soc. 2008;14(6):933-46.

69. Stanfield AC, McIntosh AM, Spencer MD, Philip R, Gaur S, Lawrie SM. Towards a neuroanatomy of autism: a systematic review and meta-analysis of structural magnetic resonance imaging studies. Eur Psychiatry. 2008;23(4):289-99.

70. Vissers ME, Cohen MX, Geurts HM. Brain connectivity and high functioning autism: a promising path of research that needs refined models, methodological convergence, and stronger behavioral links. Neurosci Biobehav Rev. 2012;36(1):604-25.

71. Rudie JD, Brown JA, Beck-Pancer D, Hernandez LM, Dennis EL, Thompson $\mathrm{PM}$, et al. Altered functional and structural brain network organization in autism. Neurolmage Clin. 2012;2:79-94.

72. Uddin LQ, Supekar K, Menon V. Reconceptualizing functional brain connectivity in autism from a developmental perspective. Front Hum Neurosci. 2013;7:458

73. Zhan Y, Paolicelli RC, Sforazzini F, Weinhard L, Bolasco G, Pagani F, et al. Deficient neuron-microglia signaling results in impaired functional brain connectivity and social behavior. Nat Neurosci. 2014;17(3):400-6.

74. Missler M, Zhang W, Rohlmann A, Kattenstroth G, Hammer RE, Gottmann K, et al. Alpha-neurexins couple Ca2+ channels to synaptic vesicle exocytosis. Nature. 2003;423(6943):939-48.

75. Alba-Ferrara LM, de Erausquin GA. What does anisotropy measure? Insights from increased and decreased anisotropy in selective fiber tracts in schizophrenia. Front Integr Neurosci. 2013;7:9.

76. Minshew NJ, Williams DL. The new neurobiology of autism: cortex, connectivity, and neuronal organization. Arch Neurol. 2007;64(7):945-50.

77. Andreae LC, Burrone J. Spontaneous neurotransmitter release shapes dendritic arbors via long-range activation of NMDA receptors. Cell Rep. 2015;10(6):873-82.

78. Ye L, Allen WE, Thompson KR, Tian Q, Hsueh B, Ramakrishnan C, et al. Wiring and molecular features of prefrontal ensembles representing distinct experiences. Cell. 2016;165(7):1776-88.

79. Jones DK. The effect of gradient sampling schemes on measures derived from diffusion tensor MRI: a Monte Carlo study. Magn Reson Med. 2004; 51(4):807-15. 